Case Report

\title{
SOX5-Null Heterozygous Mutation in a Family with Adult-Onset Hyperkinesia and Behavioral Abnormalities
}

\author{
Michael Zech, ${ }^{1,2}$ Katharina Poustka, ${ }^{3}$ Sylvia Boesch, ${ }^{4}$ Riccardo Berutti, ${ }^{5}$ Tim M. Strom, ${ }^{5,6}$ \\ Wolfgang Grisold, ${ }^{3}$ Werner Poewe, ${ }^{4}$ and Juliane Winkelmannn ${ }^{1,2,6,7}$ \\ ${ }^{1}$ Institut für Neurogenomik, Helmholtz Zentrum München, Munich, Germany \\ ${ }^{2}$ Klinik und Poliklinik für Neurologie, Klinikum rechts der Isar, Technische Universität München, Munich, Germany \\ ${ }^{3}$ Kaiser-Franz-Josef-Spital Wien, Vienna, Austria \\ ${ }^{4}$ Department of Neurology, Medical University Innsbruck, Innsbruck, Austria \\ ${ }^{5}$ Institut für Humangenetik, Helmholtz Zentrum München, Munich, Germany \\ ${ }^{6}$ Institut für Humangenetik, Technische Universität München, Munich, Germany \\ ${ }^{7}$ Munich Cluster for Systems Neurology, SyNergy, Munich, Germany \\ Correspondence should be addressed to Juliane Winkelmann; juliane.winkelmann@tum.de
}

Received 24 July 2017; Accepted 13 September 2017; Published 29 October 2017

Academic Editor: Christos Yapijakis

Copyright (C) 2017 Michael Zech et al. This is an open access article distributed under the Creative Commons Attribution License, which permits unrestricted use, distribution, and reproduction in any medium, provided the original work is properly cited.

\begin{abstract}
SOX5 encodes a conserved transcription factor implicated in cell-fate decisions of the neural lineage. SOX5 haploinsufficiency induced by larger genomic deletions has been linked to a recognizable pediatric syndrome combining developmental delay with intellectual disability, mild dysmorphism, inadequate behavior, and variable additional features including motor disturbances. In contrast to SOX5-involving deletions, examples of pathogenic SOX5 small coding variations are sparse in the literature and have been described only in singular cases with phenotypic abnormalities akin to those seen in the SOX5 microdeletion syndrome. Here a novel SOX5 loss-of-function point mutation, c.13C>T (p.Arg5X), is reported, identified in the course of exome sequencing applied to the diagnosis of an unexplained adult-onset motor disorder. Aged 43 years, our female index patient demonstrated abrupt onset of mixed generalized hyperkinesia, with dystonic and choreiform movements being the most salient features. The movement disorder was accompanied by behavioral problems such as anxiety and mood instability. The mutation was found to be inherited to the patient's son who manifested abnormal behavior including diminished social functioning, paranoid ideation, and anxiety since adolescence. Our results expand the compendium of SOX5 damaging single-nucleotide variation mutations and suggest that SOX5 haploinsufficiency might not be restrictively associated with childhood-onset syndromic disease.
\end{abstract}

\section{Introduction}

SOX5, which codes for the transcription factor SOX-5 on chromosome 12p12.1, is a pivotal regulator of gene-expression profiles during neurogenesis and thus essential in preserving nervous system integrity [1]. A role for SOX5 haploinsufficiency in the manifestation of genetic disease in humans was first appreciated by Lamb and colleagues in a 2012 publication [2], describing monoallelic perturbation of SOX5 in 16 individuals with a neurodevelopmental disorder characterized by intellectual disability, speech delay, dysmorphia, behavior problems, and variable other symptoms including motor dysfunction (referred to as Lamb-Shaffer syndrome,
MIM616803). In these original patients, haploinsufficiency of SOX5 was induced by DNA structural variations, mostly microdeletions (SOX5 partial or full gene deletions), in the 12 p12.1 chromosomal region, as demonstrated by array comparative genomic hybridization diagnostics. Several subsequent studies reporting on the identification of independent patients with deletions encompassing SOX5 confirmed these initial findings and contributed to the definition of SOX5related disease as a multisystemic pediatric syndrome [35]. More recently, the first intragenic SOX5 point mutation, a unique pathogenic stop-gain alteration, was detected by whole-exome sequencing (WES) in a patient featuring 
relevant phenotypic overlap with the SOX5 microdeletion patients [6]. Finally, in the search for novel molecular factors underlying intellectual disability, a 2016 large-scale trio-based whole-exome study discovered significantly more SOX5 lossof-function coding mutations than expected among $\sim 2,100$ affected individuals [7]. These patients were reported to have intellectual disability with additional features of language delay, facial dysmorphic signs, and behavior deficits, consistent with the previously delineated SOX5 haploinsufficiency phenotype. Although the genetic and clinical determinants of this newly identified syndrome have begun to be elucidated, our understanding of the SOX5 mutational landscape as well as the entire phenotype spectrum linked to SOX5 haploinsufficiency is incomplete and awaits further investigation. In this report, we extend the role of heterozygous SOX5 deficiency in human disease by describing an Austrian family with an index patient primarily enrolled in a study of the genetic aetiology of an adult-onset movement disorder.

\section{Case Presentations}

The index patient, individual II-2 in Figure 1(a), was a 47year-old woman of Austrian descent. She had an older, healthy brother (individual II-1). Family history was unremarkable on both the maternal and paternal side. The index patient first developed gait clumsiness and adventitious movements of the trunk and arms while walking at the age of 43 years. These symptoms were relatively sudden in onset (days) but the patient was unaware of any triggering factor. Over the next few months, the patient progressively manifested continuous abnormal involuntary movements affecting her face, neck, trunk, and all 4 extremities. Concurrently, she noted intermittent worsening of lower-limb incoordination. She reported impairment of fine motor tasks, with dropping of objects when under psychological stress. At this time, she consulted several local medical services and neurologists who interpreted her condition as "idiopathic choreo-dystonic syndrome" or "generalized dyskinesia of unknown origin." Given her acute presentation of motor impairment, a functional movement disorder was also discussed but deemed unlikely because of the lack of additional signs and symptoms characteristic of a psychogenic illness [8]. Extensive routine diagnostic work-up including systematic blood chemical analysis, screening for acanthocytes, laboratory testing for Wilson's disease, panel testing for autoimmune-encephalitis antibodies, antibody screening for neurotropic viruses, CSF analysis, electroencephalography, and brain MRI was unrevealing. Genetic testing for Huntington's disease was normal. Levodopa administration was unbeneficial. Aged 45 years, the patient was referred for reevaluation of her movement disorder to our department. The neurological examination revealed cervical dystonia, truncal dystonia, dystonic finger posturing, and severe orofacial dyskinesia. There were diffuse generalized choreiform movements, more pronounced in her shoulders and arms compared to lower limbs. She displayed unsteady gait with trunk instability secondary to irregular unpredictable movements but she could walk unaided. The remainder of the examination was unremarkable. No cerebellar pathological signs, pyramidal tract abnormalities, sensory involvement, or autonomic involvement were observed. Visual acuity and hearing were normal, as were extraocular movements, deep tendon reflexes, and flexor-plantar-responses. Moreover, no systemic pathological features were evident. The patient's history was uninformative in terms of delivery and development of motor and intellectual milestones. She had attended a regular school with only minor learning disability. Her history was, however, significant for an episode of anxiety and mood swings. Between the ages of 30 and 35 years, she was treated with selective serotonin reuptake inhibitors to manage her mood disorder, which was controlled since then. Notably, there was no medication regimen with neuroleptics or any other antidopaminergic agents prior to the appearance of her hyperkinesia and therefore a pharmacological effect explaining the movement disorder cannot be suspected. At present, more than 4 years into her motor disease, the patient continued to have persistent mixed generalized hyperkinesia with pronounced dystonic and choreic elements. Symptoms were unremitting, while the patient was started on tetrabenazine with some subjective improvements in motor capabilities.

The patient had 2 children, an asymptomatic daughter (individual III-2) and an older, phenotypically abnormal son (individual III-1). The son was a 27-year-old man who was born at term after an uncomplicated pregnancy. His developmental milestones were achieved normally. Although there was no evidence of cognitive decline, he required extratutoring at school and was described as showing oppositional reactions and aggression toward his peers. He first came to medical attention at the age of 20 years due to the presentation of paranoid beliefs and difficulties in executive functioning. According to her mother, he also experienced significant anxiety. During his visit at our department at the age of 26 years, physical examination was performed without any substantial findings. In particular, there were no peculiar facial characteristics or abnormal hyperkinetic movements.

\section{Materials and Methods}

To explore the genetic underpinnings of disease in the Austrian family, we collected peripheral blood samples of the index patient II-2, her mother I-2, her son III1 , and her daughter III-2 after obtaining informed consent under institutional-review-board-approved protocols. Genomic DNA was extracted using standard procedures. We conducted WES from DNA of the index patient by using the SureSelect Human All Exon $50 \mathrm{Mb}$ Kit (v.5, Agilent Technologies) for the generation of capture libraries. Massively parallel sequencing was done on a HiSeq2500 next-generation sequencing instrument (Illumina) in paired-end (100-bp) mode with a median read depth of 185 -fold. Data were aligned to a modified human reference genome (hg19/GRCh37) and high-confidence variant calls were produced as previously described [9]. Subsequently, nonsilent variants (coding excepting nonsplicing synonymous and splicing mutations) were retained when their minor allele frequency was $<0.001$ in $~ 60,000$ exomes from the Exome Aggregation Consortium (ExAC) Browser and an in-house dataset of 10,000 ethnically matched exome-sequenced individuals. Next, filtered 


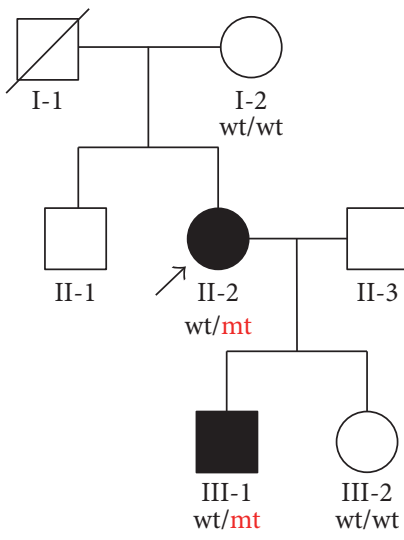

wt: wild-type mt: SOX 5 c.13C > T (p.Arg5X)

(a)
I-2

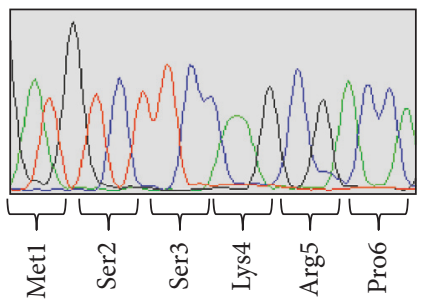

III-1

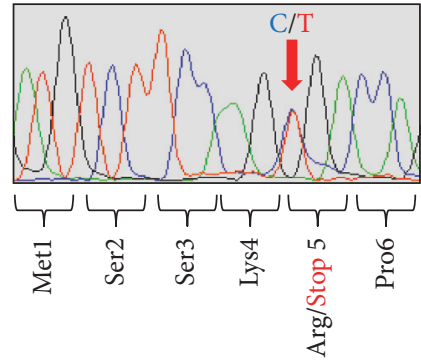

II-2 (index patient)

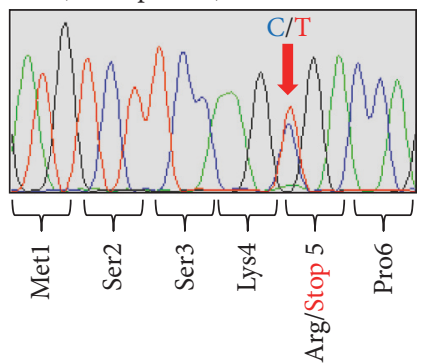

III-2

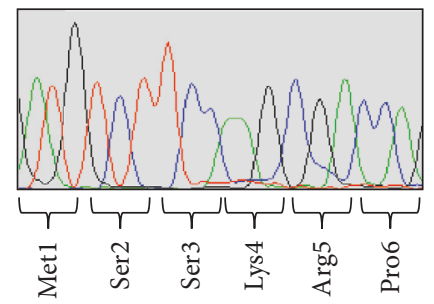

(b)

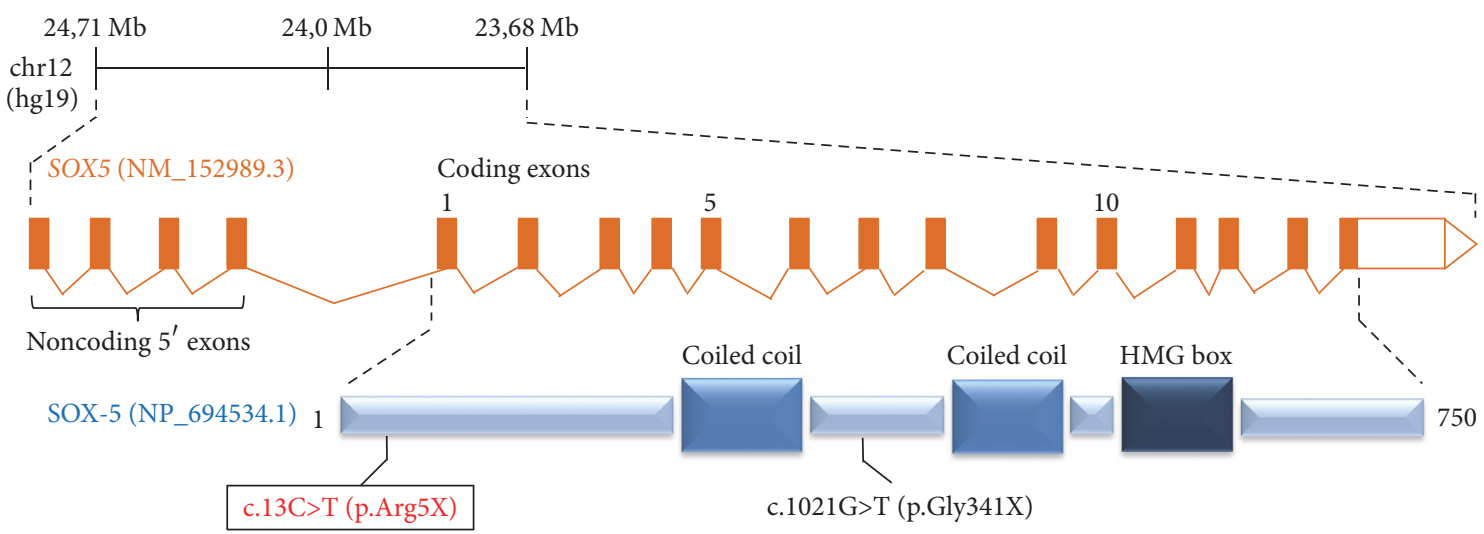

(c)

FIGURE 1: Loss-of-function short coding mutations in SOX5. (a) Pedigree of the family investigated in this study. SOX5 mutational status is shown below each tested family member. Symbols are as follows: circles, females; squares, males; filled, phenotypically abnormal; empty, healthy; slash, deceased. (b) Dideoxy sequencing traces document SOX5 c.13C > T (p.Arg5X) in genomic DNA of the index patient II-2 and her son III-1. Individuals I-2 and III-2 show homozygous wild-type sequence at this site. Arrows indicate the mutant nucleotide positions. (c) Schematic overview of the human SOX5 locus (NM_152989.3; top) and its encoded protein (NP_694534.1; bottom) illustrates the newly identified c.13C > T (p.Arg5X) stop-gain variant (boxed) and a loss-of-function point mutation previously described [6]. Notably, all of the critical SOX-5 protein domains are distal to the $\mathrm{p}$.Arg5X protein-truncation site and thus the truncated protein, if expressed, is highly unlikely to retain residual function. The schematic is simplified and not drawn to exact scale.

variants were considered to be disease-related if they resided in genes previously associated with key clinical symptoms observed in the index patient. To this end, we interrogated candidate gene lists obtained from the scientific literature [9]. These lists were curated manually and created via exhaustive phenotype-based searches (analytical phenotype search keywords: "dystonia"; "chorea"; "hyperkinesia"; "dyskinesia"; "clumsiness") in original publications provided by PubMed (https://www.ncbi.nlm.nih.gov/pubmed/) and website entries provided by the Online Mendelian Inheritance in Man (OMIM) database (https://www.omim.org/).
Dideoxy sequencing was performed to confirm the variant selected as potentially relevant to disease and test for genotype-phenotype cosegregation in the family. Amplification primers were designed with ExonPrimer (https://ihg .helmholtz-muenchen.de/ihg/ExonPrimer.html).

\section{Results}

Phenotypically guided filtering of the index patient's WES data left a single heterozygous, predicted detrimental singlenucleotide variant in SOX5, a gene previously linked to 
the phenotypes hyperkinesia and clumsiness. At nucleotide position 13 of the SOX5 brain-expressed long transcript (NM_152989.3; coding exon 1; Figure 1(c)), we identified a Cto-T substitution leading to the insertion of a premature stop codon at amino acid 5 (p.Arg5X). As a result, this variation was expected to either trigger nonsense-mediated messengerRNA decay or give rise to a truncated SOX-5 that is highly unlikely to retain functionality (Figure 1(c)). SOX5 c.13C > T (p.Arg5X) was therefore presumed to be a null allele, resulting in the inactivation of one SOX 5 copy in the index patient. The variant was absent from $\sim 60,000$ population controls in ExAC and $~ 10,000$ internal control individuals sequenced by exome. It was referenced in dbSNP142 (rs780962501) with 1 occurrence in the Genome Aggregation Database (gnomAD). The mutation was verified through dideoxy sequencing in the index patient using primers flanking the mutated base and shown to be present in her phenotypically abnormal son (III-1) (Figure 1(b)). The index patient's mother (I-2) and daughter (III-2) were each found to be homozygous for the reference allele.

\section{Discussion}

SOX5 encodes a member of the SRY-HMG-box- (SOX-) containing family of evolutionary conserved transcription factors involved in fundamental developmental pathways across multiple tissues [10]. SOX-family genes are subdivided into 10 categories (A-J), in which SOX5, along with SOX6 and SOX13, belongs to the SOXD group [10]. Expression of the long SOX5 transcript (NM_152989.3) was found to be abundant in human and mouse brain from early embryonic stages [11]. Significant amounts of past work have gone into the role of SOX5 in the maturation of the central nervous system, demonstrating that the gene is vital to the modulation of critical fate decisions, proliferation, and differentiation of various neural progenitors $[1,12,13]$. Mice with two SOX5 null alleles die in the early postnatal period [14] and their brains show severe defects in the generation of neuronal subcortical projections [12]. Similarly, a recent transgenic Drosophila model of SOX5 underscored its critical role in giving rise to proper neuronal network formation [15]. Considering the developmental significance of SOX5, it is not surprising that SOX5 dosage-reduction in humans is not tolerated and that heterozygous loss-of-function mutations in SOX5 are relevant to human disease. These suppositions are also well reflected in statistical measures gained by large population-based variation resources, such as ExAC, indicating that SOX5 is under intense selective constraint for loss-of-function alterations (probability of being loss-offunction intolerant [pLI] score 1.0) [16]. In prior literature, multiple genomic structural variations disrupting SOX5 have been described in patients but only a highly restricted number of pathogenic short intragenic mutations have been recognized. By means of WES, we detected here an additional SOX5 stop-gain variant (c.13C $>\mathrm{T}[\mathrm{p} . \operatorname{Arg} 5 \mathrm{X}]$ ), raising to two the number of detailed clinical reports on individuals with deleterious SOX 5 coding mutations. The fact that the $c .13 \mathrm{C}>\mathrm{T}$ (p.Arg5X) allele is annotated in dbSNP142 and found once in the gnomAD Browser does not conflict with the hypothesis of a clinically relevant mutation given that (1) the dbSNP database is known to contain rare pathogenic variants and (2) gnomAD includes sequence information from phenotypically abnormal individuals $[16,17]$. In particular, gnomAD incorporates data of trio sequencing performed on families with neuropsychiatric disease, a central phenotypic aspect observed in the current patients. Although SOX5 c.13C > T (p.Arg5X) was identified in the index patient's WES dataset via a clinically driven filtering strategy using the phenotype search keywords "hyperkinesias" and "clumsiness"-2 symptoms previously correlated with heterozygous SOX5 deficiency-the clinical courses observed in our motherson pair differed from those previously reported in association with SOX5 haploinsufficiency. The index patient was ascertained in the adult neurology setting with a delayedonset movement disorder, whereas the previously described patients manifested a constellation of deficits characteristic of a pediatric neurodevelopmental syndrome [2]. Likewise, the index patient's son showed no evidence of developmental or intellectual regression but presented with severe behavioral abnormalities throughout adolescence. Thus, our results indicate that the $S O X 5$-related phenotype extends well beyond childhood-onset syndromic disease. Of note, SOX5 has also been demonstrated to play an important role during chondrogenesis [10], but there were no skeletal deformities or morphological stigmata compatible with cartilage defects in the SOX5 disease subjects described here.

We note that we do not here provide unambiguous evidence to attribute the patients' SOX5 stop-gain mutations to their phenotypes, but nevertheless there are strong arguments in favor of a causal relationship: (i) from a genetics viewpoint, transcripts generated by SOX 5 c.13C $>$ T (p.Arg5X), regardless of whether they are subjected to nonsense-mediated decay or evade this mechanism, are highly likely to be nonfunctional (Figure 1(c)). Thus, c.13C > T (p.Arg5X) is a predicted null allele in a haploinsufficient gene. Furthermore, c.13C > T (p.Arg5X) was exclusive to phenotypically abnormal individuals in comparison to the rest of the family and we were unable to identify any other variant in the index patient's WES data that accounted for her phenotype; (ii) from a phenotypic viewpoint, the index patient and her son shared some core clinical features with previously described SOX5-haploinsufficient patients [2]. In fact, hyperkinesia and other types of motor dysfunction were recurrent, albeit variable findings among described SOX5-mutated subjects, whereas behavior deficits-as evident in both the mother and her son-were almost universally present. Accordingly, the observation of a more restricted disease phenotype in the patients reported here suggests that family-specific presentations outside the more commonly seen phenotypic domains may exist in SOX5-related disease. Variable expressivity has been previously recognized between and within SOX5-mutated families and it is a very well-known phenomenon in a multitude of neurodevelopmental disorders. In this context, it is also worth mentioning that recent work has found an enrichment of SOX5 missense mutations in patients with age-related neurodegeneration, hinting at an even broader role for SOX5 in human disease phenotypes [15]. Although it remains to be determined why individual 
patients are differentially affected by SOX5 mutations, it is possible that the ultimate phenotypic outcome depends on the interaction of their biological effects with modifier genes, genetic background, and environmental exposures; (iii) from a mechanistic viewpoint, SOX5 haploinsufficiency appears to be a plausible pathophysiological event underlying the observed phenotypes. Hyperkinetic movement disorders and behavior deficits are genetically profoundly heterogeneous conditions that arise as a result of mutations in genes involved in various biologically relevant activities. For both pathologies, however, heterozygous disruption of transcription factor-encoding genes has emerged as an important disease mechanism [18, 19]. Furthermore, it is especially interesting to consider that a connection between movement disorders, in particular dystonia, and mutations of other SOX-family genes has been proposed previously by separate studies. Ebrahimi-Fakhari and colleagues reported a patient with generalized dystonia who was found to harbor a de novo heterozygous deletion in SOX6, another member of the SOXD subgroup [20]. Remarkably, reminiscent of the presentation seen in our SOX5-mutated index patient, dystonia in the SOX6 deletion patient was of acute onset and combined with other hyperkinesia. In addition, Bakrania and colleagues described lower-limb dystonia in association with a heterozygous protein-truncating mutation in SOX2 [21].

To conclude, we have identified an ultrarare, previously undescribed SOX5 loss-of-function allele in a family with adult-onset abnormal hyperkinetic movements and psychiatric disturbances, thereby suggesting an expansion of the genotypic and clinical spectrum linked to SOX5 haploinsufficiency. Future studies are warranted to further explore a possible role of SOX5 in movement and neuropsychiatric disorders and advance knowledge of the clinical heterogeneity that arises upon inactivation of one SOX5 copy in humans. Given the phenotypic variability that is indicated by the current investigation, we posit that the presence of hyperkinesia in both the context of a childhood neurodevelopmental disorder and a late-onset acute presentation should prompt a search for heterozygous SOX5 mutations in array diagnostics, exome sequencing, or genome-sequencing data. The breath of phenotypic variation related to mutations in SOX5 is likely to increase when additional patients will be identified over the coming years.

\section{Consent}

Written informed consent was obtained from all participants of the study.

\section{Conflicts of Interest}

The authors of this manuscript have no conflicts of interest to declare.

\section{References}

[1] K. Y. Kwan, M. M. S. Lam, Ž. Krsnik, Y. I. Kawasawa, V. Lefebvre, and N. Šestan, "SOX5 postmitotically regulates migration, postmigratory differentiation, and projections of subplate and deep-layer neocortical neurons," Proceedings of the National Acadamy of Sciences of the United States of America, vol. 105, no. 41, pp. 16021-16026, 2008.

[2] A. N. Lamb, J. A. Rosenfeld, N. J. Neill et al., "Haploinsufficiency of SOX5 at 12p12.1 is associated with developmental delays with prominent language delay, behavior problems, and mild dysmorphic features," Human Mutation, vol. 33, no. 4, pp. 728740, 2012.

[3] R. W. Y. Lee, J. Bodurtha, J. Cohen, A. Fatemi, and D. Batista, "Deletion 12p12 involving SOX5 in two children with developmental delay and dysmorphic features," Pediatric Neurology, vol. 48, no. 4, pp. 317-320, 2013.

[4] I. Schanze, D. Schanze, C. A. Bacino, S. Douzgou, B. Kerr, and M. Zenker, "Haploinsufficiency of SOX5, a member of the SOX (SRY-related HMG-box) family of transcription factors is a cause of intellectual disability," European Journal of Medical Genetics, vol. 56, no. 2, pp. 108-113, 2013.

[5] I. Quintela, F. Barros, R. Lago-Leston, M. Castro-Gago, A. Carracedo, and J. Eiris, "A maternally inherited 16p13.11-p12.3 duplication concomitant with a de novo SOX5 deletion in a male patient with global developmental delay, disruptive and obsessive behaviors and minor dysmorphic features," American Journal of Medical Genetics Part A, vol. 167, no. 6, pp. 1315-1322, 2015.

[6] A. Nesbitt, E. J. Bhoj, K. McDonald Gibson et al., "Exome sequencing expands the mechanism of SOX5-associated intellectual disability: a case presentation with review of sox-related disorders," American Journal of Medical Genetics Part A, vol. 167, no. 11, pp. 2548-2554, 2015.

[7] S. H. Lelieveld, M. R. F. Reijnders, R. Pfundt et al., "Metaanalysis of 2,104 trios provides support for 10 new genes for intellectual disability," Nature Neuroscience, vol. 19, no. 9, pp. 1194-1196, 2016.

[8] M. J. Edwards and K. P. Bhatia, "Functional (psychogenic) movement disorders: merging mind and brain," The Lancet Neurology, vol. 11, no. 3, pp. 250-260, 2012.

[9] M. Zech, S. Boesch, A. Jochim et al., "Clinical exome sequencing in early-onset generalized dystonia and large-scale resequencing follow-up," Movement Disorders, vol. 32, no. 4, pp. 549-559, 2017.

[10] Y. Kamachi and H. Kondoh, "Sox proteins: regulators of cell fate specification and differentiation," Development, vol. 140, no. 20, pp. 4129-4144, 2013.

[11] T. Ikeda, J. Zhang, T. Chano et al., "Identification and characterization of the human long form of Sox5 (L-SOX5) gene," Gene, vol. 298, no. 1, pp. 59-68, 2002.

[12] T. Lai, D. Jabaudon, B. J. Molyneaux et al., "SOX5 controls the sequential generation of distinct corticofugal neuron subtypes," Neuron, vol. 57, no. 2, pp. 232-247, 2008.

[13] P. L. Martinez-Morales, A. C. Quiroga, J. A. Barbas, and A. V. Morales, "SOX5 controls cell cycle progression in neural progenitors by interfering with the WNT-B-catenin pathway," EMBO Reports, vol. 11, no. 6, pp. 466-472, 2010.

[14] C. P. Hersh, E. K. Silverman, J. Gascon et al., "SOX5 is a candidate gene for chronic obstructive pulmonary disease susceptibility and is necessary for lung development," American Journal of Respiratory and Critical Care Medicine, vol. 183, no. 11, pp. 1482-1489, 2011.

[15] A. Li, B. Hooli, K. Mullin et al., "Silencing of the Drosophila ortholog of SOX5 leads to abnormal neuronal development and behavioral impairment," Human Molecular Genetics, vol. 26, no. 8, pp. 1472-1482, 2017. 
[16] M. Lek, K. J. Karczewski, E. V. Minikel et al. et al., "Analysis of protein-coding genetic variation in 60,706 humans," Nature, vol. 536, no. 7616, pp. 285-291, 2016.

[17] W. Song, S. A. Gardner, H. Hovhannisyan et al., "Exploring the landscape of pathogenic genetic variation in the ExAC population database: insights of relevance to variant classification," Genetics in Medicine, vol. 18, no. 8, pp. 850-854, 2016.

[18] K. Lohmann and C. Klein, "Update on the genetics of dystonia," Current Neurology and Neuroscience Reports, vol. 17, no. 3, article 26, 2017.

[19] Deciphering Developmental Disorders S, "Large-scale discovery of novel genetic causes of developmental disorders," Nature, vol. 519, pp. 223-228, 2015.

[20] D. Ebrahimi-Fakhari, B. Maas, C. Haneke et al., "Disruption of SOX6 is associated with a rapid-onset dopa-responsive movement disorder, delayed development, and dysmorphic features," Pediatric Neurology, vol. 52, no. 1, pp. 115-118, 2015.

[21] P. Bakrania, D. O. Robinson, D. J. Bunyan et al., "SOX2 anophthalmia syndrome: $12 \mathrm{New}$ cases demonstrating broader phenotype and high frequency of large gene deletions," British Journal of Ophthalmology, vol. 91, no. 11, pp. 1471-1476, 2007. 


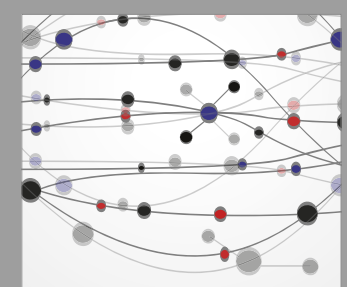

The Scientific World Journal
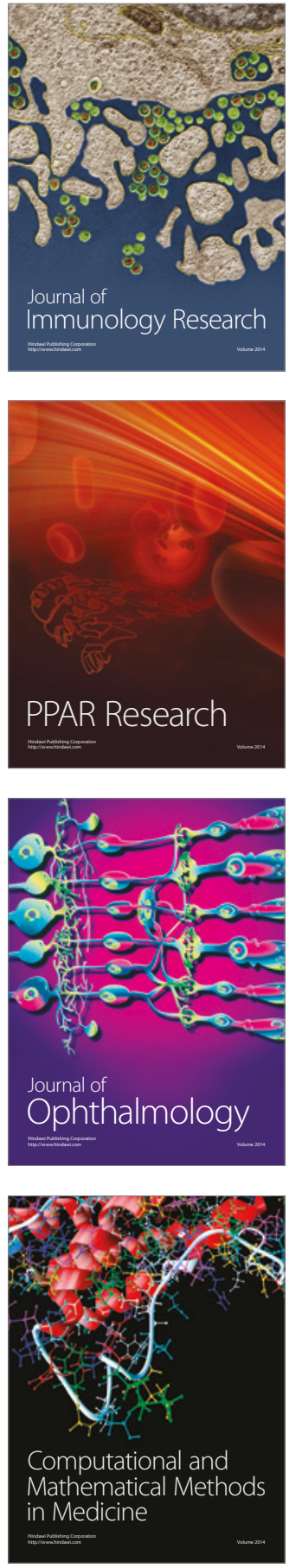

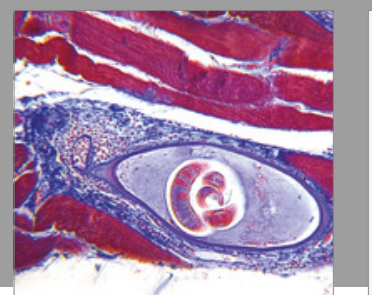

Gastroenterology Research and Practice
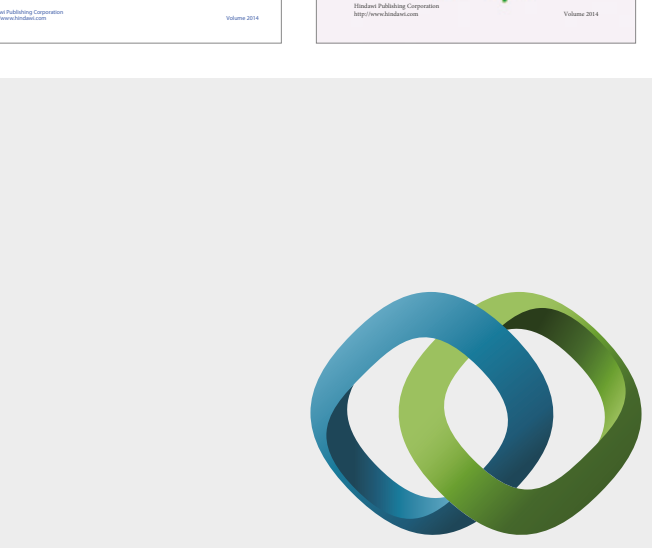

\section{Hindawi}

Submit your manuscripts at

https://www.hindawi.com
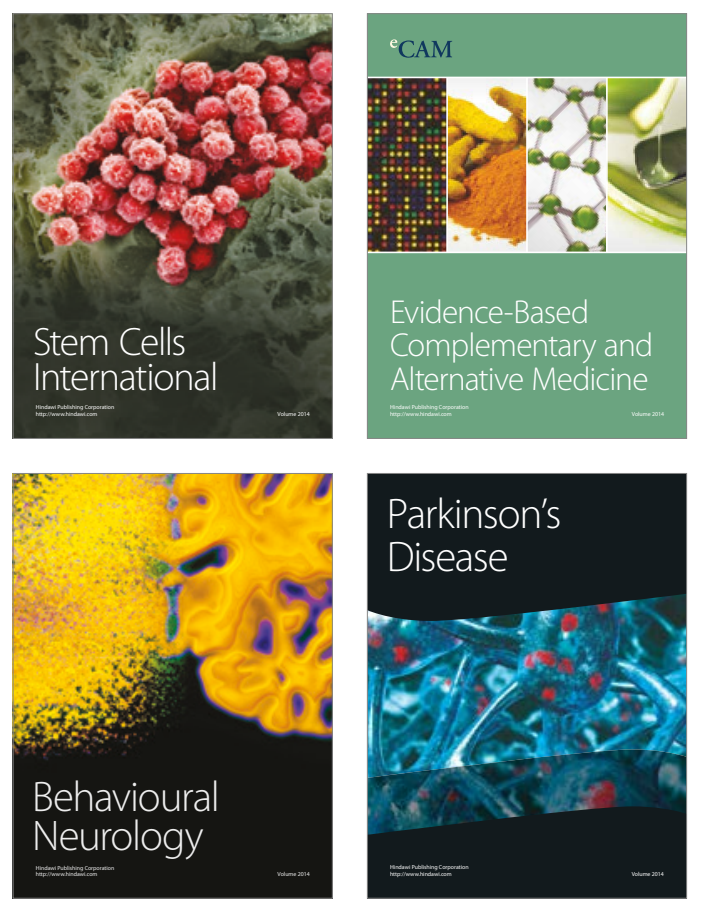
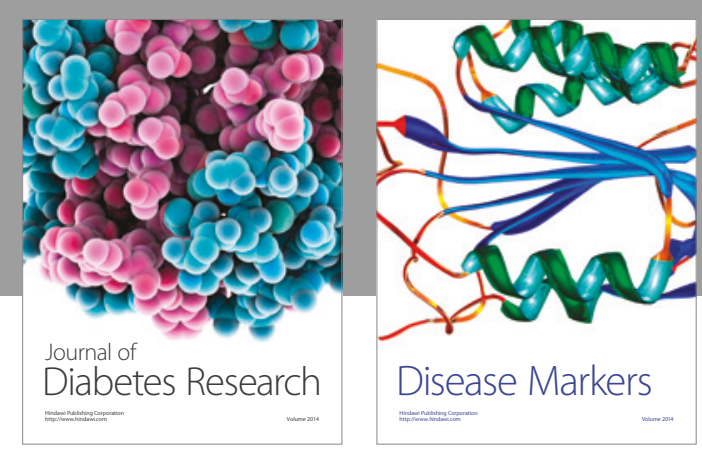

Disease Markers
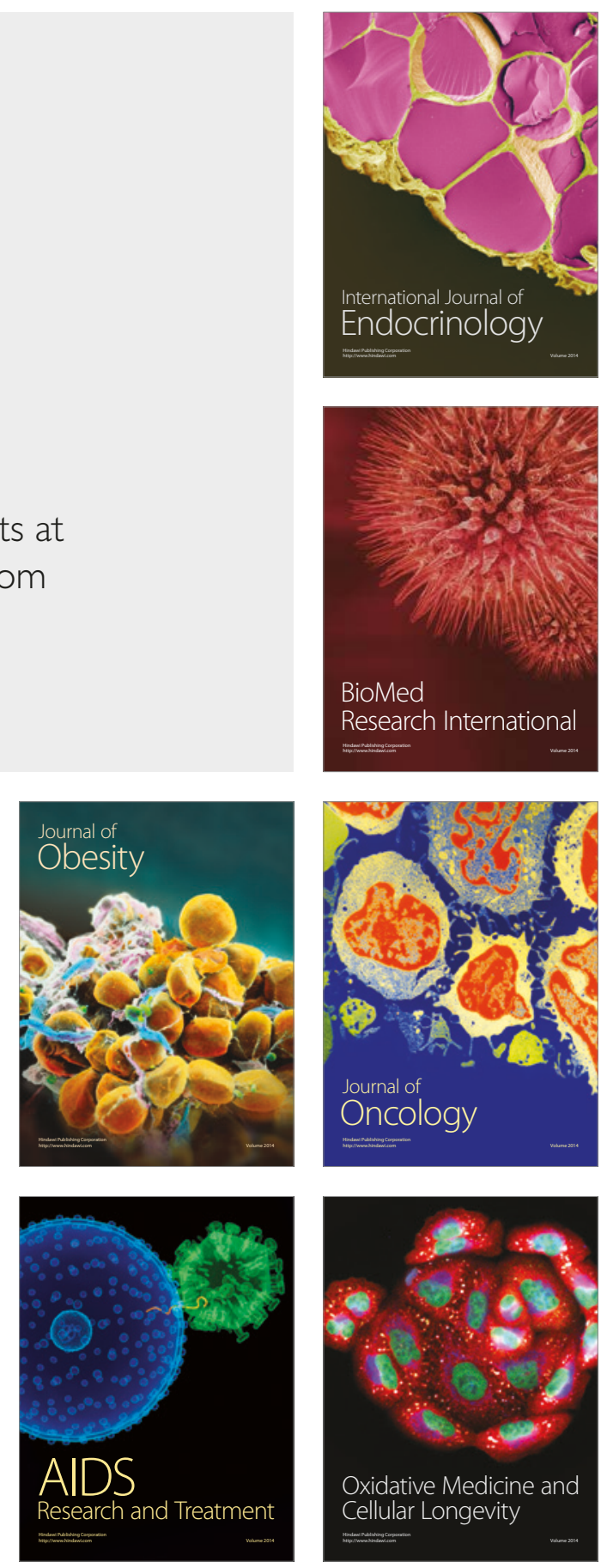